\title{
Brown-York quasilocal energy in Lanczos-Lovelock gravity and black hole horizons
}

\author{
Sumanta Chakraborty ${ }^{a, 1}$ and Naresh Dadhich ${ }^{b, a}$ \\ ${ }^{a}$ IUCAA, \\ Post Bag 4, Ganeshkhind, Pune University Campus, Pune 411 007, India \\ ${ }^{b}$ Center for Theoretical Physics, Jamia Millia Islamia, \\ New Delhi 110025, India \\ E-mail: sumanta@iucaa.in, nkd@iucaa.ernet.in
}

\begin{abstract}
A standard candidate for quasilocal energy in general relativity is the BrownYork energy, which is essentially a two dimensional surface integral of the extrinsic curvature on the two-boundary of a spacelike hypersurface referenced to flat spacetime. Several years back one of us had conjectured that the black hole horizon is defined by equipartition of gravitational and non-gravitational energy. By employing the above definition of quasilocal Brown-York energy, we have verified the equipartition conjecture for static charged and charged axi-symmetric black holes in general relativity. We have further generalized the Brown-York formalism to all orders in Lanczos-Lovelock theories of gravity and have verified the conjecture for pure Lovelock charged black hole in all even $d=2 m+2$ dimensions, where $m$ is the degree of Lovelock action. It turns out that the equipartition conjecture works only for pure Lovelock, and not for Einstein-Lovelock black holes.
\end{abstract}

KEywords: Classical Theories of Gravity, Black Holes

ARXIV EPRINT: 1509.02156

${ }^{1}$ Corresponding author. 


\section{Contents}

1 Introduction

2 Brown-York quasilocal energy and black hole horizons in general relativity

2.1 Brown-York quasilocal energy in an arbitrary static spherically symmetric spacetime

2.2 Kerr-Newmann black hole

3 Brown-York quasilocal energy and black hole horizons in LanczosLovelock gravity

3.1 Brown-York quasilocal energy in Lanczos-Lovelock gravity: formalism

3.2 Brown-York quasilocal energy in Lanczos-Lovelock gravity and equipartition 12

3.3 Equipartition conjecture in Einstein-Gauss-Bonnet gravity

\section{Introduction}

Defining quasilocal energy for general relativity is an extremely important but a long eluding problem. Initial attempts in this direction involved pseudotensor methods, leading to coordinate dependent expressions, identification of certain symmetries and defining the Noether charge or some mathematical constructs from the Cauchy data showing similar physical properties associated with energy. Most of these definitions are quite useful and have physical implications in one case or the other, but no unified description has emerged (for a partial set of references see [1-7]).

The main reason for this ambiguity in defining an energy for gravitational field is due to its non-linear nature and the fact that gravitational energy is non-localizable. This in turn implies not only matter energy produces gravitational field but the gravitational field energy also does the same. A natural definition of quasilocal gravitational energy follows from employing the Hamilton-Jacobi theory for gravitation. This leads to the Brown-York quasilocal energy as it was first obtained by Brown and York in [8]. This definition of energy contains, as it should, contributions from both the matter part and the gravity part. It is defined covariantly and more importantly the energy is additive. Also in the asymptotic limit the Brown-York energy reproduces the Arnowitt-Deser-Misner mass, providing much credence to it. This quasilocal energy has been studied extensively in general relativity for static spacetime with spherical symmetry and also for Kerr black hole, which is stationary [9-12]. 
One key aspect for general relativity is that, it simply follows from geometric properties of the Riemann curvature tensor, in particular the Bianchi identity plays a crucial role. Moreover the field equations for general relativity is second order in the dynamical variable, which ensures that ghost modes do not appear. Then it is interesting to ask whether this setup can be generalized to higher dimensions as well as to higher curvature theories. Remarkably the answer is yes. If we demand that field equations have to be second order in the dynamical variable, the gravitational Lagrangian uniquely picks out the Lanczos-Lovelock action [13-17]. On the other hand, generalizing the curvature tensor such that trace of its Bianchi derivative vanishes giving the divergence free second rank tensor, which agrees with the one obtained by variation of the action. This also uniquely leads to the LanczosLovelock Lagrangian [18]. The pure Lanczos-Lovelock Lagrangian is also intimately connected with spacetime dimensions. For example, field equations for general relativity is nontrivial for $D>2$ and has free propagation only in $D>3$. For $D=3$, gravity is kinematic, as Riemann tensor is determined entirely by Ricci tensor. Then requiring the kinematic property of gravity to hold in all odd dimensions singles out pure Lanczos-Lovelock gravity, i.e., one particular order out of the full Lanczos-Lovelock Lagrangian [19-22]. Moreover, from thermodynamic perspectives as well Lanczos-Lovelock gravity has unique features [23]. Most of the thermodynamic results which hold for null surfaces in general relativity can be generalized to hold in Lanczos-Lovelock gravity as well [24-28]. Thus even from the thermodynamic perspective, Lanczos-Lovelock gravity has a special status. As argued earlier, the quasilocal energy is an important measure of gravitational field, it would therefore be pertinent to study the Brown-York quasilocal energy for Lanczos-Lovelock gravity as well.

In general, location of black hole horizon is defined as a limit for timelike world lines to exist (when 4-velocity turns null) and when a spatial surface turns one way membrane (i.e., it can be crossed in one direction only). It has been argued in [10] that at the black hole horizon timelike particles (that are pulled by gradient of potential produced by matter (nongravitational) energy) tend to photons that can feel no gravitational pull but only follow curvature of space produced by gravitational field energy. The horizon should therefore be defined when their respective sources are equal in magnitude. That is equipartition between gravitational and non-gravitational (matter) energy. By using Brown-York energy, gravitational field energy was computed and the equipartition was shown to exist for static black hole horizons. This is the conjecture for location of black hole horizon which we would like to examine for stationary black holes in general relativity as well as with the appropriate generalization of Brown-York energy for static black holes in Lanczos-Lovelock gravity. It has been previously tested for static black holes in general relativity [10]. In this work our main motivation, besides obtaining the quasilocal energy for Lanczos-Lovelock gravity, is to test the veracity of this equipartition conjecture for stationary black holes in general relativity and static black holes in Lanczos-Lovelock gravity.

The paper is organized as follows, in section 2 we discuss the Brown-York energy in the context of general relativity and then we have applied it to Reissner-Nordström and KerrNewmann black holes to obtain the location of the horizon through equipartition. Then in the subsequent section, i.e., in section 3 we have discussed extensively the quasilocal energy for Lanczos-Lovelock gravity and equipartition conjecture in it. Finally we conclude with a short discussion on our results. 
In this paper we will work with the $(-,+,+, \ldots)$ signature for the spacetime metric and shall set the fundamental constants $G$ and $c$ to unity.

\section{Brown-York quasilocal energy and black hole horizons in general rel- ativity}

We will start with a spacetime region $\mathcal{M}$, which is topologically $\Sigma$ times a real line interval, where $\Sigma$ is the three-space. The boundary of the three-space $\Sigma$, is denoted by $\mathcal{B}$ (not necessarily simply connected), which is two dimensional. The product of $\mathcal{B}$ with timelike world lines normal to $\Sigma$ is denoted by $B$ and the end points of the timelike world lines are denoted by $\Sigma_{1}$ and $\Sigma_{2}$. Hence $B, \Sigma_{1}$ and $\Sigma_{2}$ form the three-boundary of the full spacetime region $\mathcal{M}$.

The full spacetime metric is $g_{a b}, u_{a}$ is the future pointing timelike normal to the hypersurface $\Sigma$, and $n_{a}$ is the spacelike normal to the three-boundary $B$. The metric and extrinsic curvature on $\Sigma$ are denoted by $h_{a b}$ and $K_{a b}$ respectively, with $h_{b}^{a}$ acting as the projection tensor on $\Sigma$. From hypersurface orthogonality between $\Sigma$ and $B$ it immediately follows that $u_{a} n^{a}=0$. Similar projection tensors and extrinsic curvatures can be defined on $B$ as well. This can be extended to finally introduce the extrinsic curvature $k_{A B}$ on the two-boundary $\mathcal{B}$ of $\Sigma$ with the induced metric, $q_{b}^{a}=\delta_{b}^{a}+u^{a} u_{b}-n^{a} n_{b}$. Given this spacetime foliation we can introduce the ADM decomposition [13, 29, 30] and thus the action can be written as a bulk term which includes intrinsic quantities defined on $\Sigma$ and a surface term on the three-boundary. On the two end points $\Sigma_{1}$ and $\Sigma_{2}$ the surface term equals $2 K$, where $K=K_{a b} h^{a b}$ is the trace of the extrinsic curvature on $\Sigma$. On the other surface, namely $B$, the surface term equals the trace of the extrinsic curvature defined on $B$. Then under variation we will obtain the gravitational momentum conjugate to both $h_{a b}$ on $\Sigma$ and the respective one on $B$. Treating the gravitational action analogously to a matter action and from the Hamilton-Jacobi method the quasilocal gravitational energy contained within the two-surface $\mathcal{B}$ turns out to be [8]

$$
E_{\mathrm{BY}}=\frac{1}{8 \pi} \int_{\mathcal{B}} d^{2} x \sqrt{q}\left(k-k_{0}\right)
$$

where $q$ is the two-metric defined on the two-surface $\mathcal{B}$ and $k_{0}$ stands for the trace of extrinsic curvature for some reference spacetime. As the two-surface $\mathcal{B}$ tends to infinity, the Brown-York energy would approach the ADM mass (the positivity of Brown-York energy and its relation to hoop conjecture have been explored in [31-34]). We will be interested in asymptotically flat solutions in which $k_{0}$ is the trace of extrinsic curvature of $\mathcal{B}$ as embedded in a flat spacetime. In this section we will evaluate the quasilocal energy for two cases: (a) static spherically symmetric and (b) stationary axially symmetric spacetimes, in particular for Reissner-Nordström and Kerr-Newmann black holes. In both these cases we will explicitly see that in the asymptotic limit the Brown-York energy goes to the ADM energy [29], which is a crucial check for the validity of any definition of energy in general relativity. 


\subsection{Brown-York quasilocal energy in an arbitrary static spherically symmetric spacetime}

We will first derive the expression for Brown-York energy for the most general static spherically symmetric spacetime [35-37] with the following line element

$$
d s^{2}=-f(r) d t^{2}+\frac{d r^{2}}{g(r)}+r^{2} d \Omega^{2}
$$

The metric is assumed to be asymptotically flat, i.e., in the limit $r \rightarrow \infty$ both $f(r)$ and $g(r)$ go to unity giving the flat Minkowskian metric. The hypersurface $\Sigma$ is taken to be a $t=$ constant hypersurface and $B$ as a $r=$ constant surface. The two-surface $\mathcal{B}$ is a $r=$ constant hypersurface within $\Sigma$. Then the unit timelike normal $u_{a}$ to $\Sigma$ and unit spacelike normal $n_{a}$ to $B$ turn out to be

$$
\begin{aligned}
u_{a} & =-\sqrt{f}(1,0,0,0) ; & u^{a} & =\frac{1}{\sqrt{f}}(1,0,0,0) \\
n_{a} & =\frac{1}{\sqrt{g}}(0,1,0,0) ; & n^{a} & =\sqrt{g}(0,1,0,0)
\end{aligned}
$$

Having obtained the normal to $\Sigma$ and $B$ we can now compute the corresponding extrinsic curvatures, and in particular for the latter we have,

$$
\begin{aligned}
k & =-\frac{1}{\sqrt{h}} \partial_{\mu}\left(\sqrt{h} n^{\mu}\right)=-\partial_{r} n^{r}-n^{r} \partial_{r} \ln \sqrt{h} \\
& =-\partial_{r} \sqrt{g}-\sqrt{g} \partial_{r} \ln \left(\frac{r^{2} \sin \theta}{\sqrt{g}}\right)=-\frac{2}{r} \sqrt{g(r)}
\end{aligned}
$$

The embedding of $\mathcal{B}$ in a flat spacetime is trivial and the trace of extrinsic curvature $k_{0}$ is simply $-2 / r$. Then using the expression for $k$ from eq. (2.5) the Brown-York energy as defined in eq. (2.1) yields,

$$
E_{\mathrm{BY}}=\frac{1}{4 \pi} \int d \theta d \phi r^{2} \sin \theta \frac{1}{r}(1-\sqrt{g(r)})=r(1-\sqrt{g(r)})
$$

This expression for Brown-York energy is completely general. Given any static, spherically symmetric spacetime in general relativity with line element given by eq. (2.2) the above result holds. This also shows that the Brown-York energy singles out the coefficient of $g_{r r}$, rather than that of $g_{t t}$. The implication of which is straightforward - the gravitational energy really resides in the spatial curvature, i.e. it curves the three space. This is in complete accord with some of the earlier studies by one of us [9].

Having derived the general expression, let us now consider an application of this result. For that we pick up the Reissner-Nordström black hole, whose metric elements are given by

$$
f(r)=g(r)=1-\frac{2 M}{r}+\frac{Q^{2}}{r^{2}}
$$

The Brown-York quasilocal energy within a sphere of radius $r_{0}$ turns out to be,

$$
E\left(r \leq r_{0}\right)=r_{0}\left[1-\sqrt{1-\frac{2 M}{r_{0}}+\frac{Q^{2}}{r_{0}^{2}}}\right]
$$


where $M$ and $Q$ respectively stand for mass and charge of the black hole. Let us expand it for large $r$ to write

$$
E\left(r \leq r_{0}\right) \approx M-\frac{Q^{2}}{2 r_{0}}+\frac{M^{2}}{2 r_{0}}
$$

Clearly it goes to the desired limit, the ADM mass M, asymptotically. It is also evident from the expression of quasi-local energy that, $\mathrm{E}$ is the sum of matter energy and potential energy associated with building a charged fluid ball by bringing together individual particles from some initial radius. It can also have the following understanding: $M$ being the total energy including rest mass and all kinds of interaction energies. The energy lying exterior to the radius $r_{0}$ will be $Q^{2} / 2 r_{0}$, arising from the energy momentum tensor component $T_{0}^{0}=Q^{2} / 2 r_{0}^{4}$ due to electric field. The contribution of gravitational potential energy corresponds to the second term in the approximation, i.e., $-M^{2} / 2 r_{0}$. Thus the energy within radius $\mathrm{R}$ will correspond to $M-\left(Q^{2} / 2 r_{0}-M^{2} / 2 r_{0}\right)$, exactly coinciding with eq. (2.9).

For extremal black hole $M=Q$, the two energies cancel out each other exactly, not only in the asymptotic expansion but everywhere. Thus alike the Komar mass for the Schwarzschild black hole, the Brown-York energy is conserved, and is equal to ADM mass, for the extremal Reissner-Nordström black hole. The energy within radius $r_{0}$ is given by the Brown-York expression as presented in eq. (2.8), while the energy outside $r_{0}$ has two parts - (a) the energy contained within the gravitational field $E_{\text {grav }}$ and (b) the energy contained within the electric field $Q^{2} / 2 r_{0}$ [10]. This separation of energy into two parts can be written as,

$$
E\left(r \geq r_{0}\right)=E_{\text {grav }}+\frac{Q^{2}}{2 r_{0}}
$$

The total energy in the full spacetime manifold corresponds to the ADM mass. This implies, $E\left(r \leq r_{0}\right)+E\left(r \geq r_{0}\right)=M$, the ADM mass. Using which the gravitational energy turns out to be

$$
E_{\text {grav }}=M-\frac{Q^{2}}{2 r_{0}}-r_{0}\left[1-\sqrt{1-\frac{2 M}{r_{0}}+\frac{Q^{2}}{r_{0}^{2}}}\right]
$$

On the other hand the non-gravitational energy outside radius $r_{0}$ arises from the energy density $Q^{2} / 2 r_{0}^{4}$ due to electric field. Integrating the electric field energy over the range of radial distance from $\infty$ to $r_{0}$, we get $-Q^{2} / 2 r_{0}$. Adding $M$ to it we obtain the nongravitational energy to be, $E_{\text {non-grav }}=M-Q^{2} / 2 r_{0}$. Now according to the equipartition conjecture, the horizon is defined when energy is equally divided between the matter fields and the gravitational field, leading to, $E_{\text {grav }}+E_{\text {non-grav }}=0$, which implies

$$
\sqrt{1-\frac{2 M}{r_{0}}+\frac{Q^{2}}{r_{0}^{2}}}\left[1-\sqrt{1-\frac{2 M}{r_{0}}+\frac{Q^{2}}{r_{0}^{2}}}\right]=0 .
$$

This clearly has two solutions, namely, (a) $r=r_{+}=M+\sqrt{M^{2}-Q^{2}}$, the larger root of $r^{2}-2 M r+Q^{2}=0$, yielding the location of the event horizon and (b) $r=Q^{2} / 2 M$, the hard core radius for naked singularity for the parameter space $Q^{2}>M^{2}$. In the latter case, note that the hard core radius marks vanishing of non-gravitational (matter) energy which consequently implies vanishing of gravitational energy as well because the 
latter is created by the former. Then energy contained inside the hard core radius also vanishes and the entire mass $M$ lies outside. The hard core radius should rather be looked upon as the radius where non-gravitational energy goes to zero rather than equipartition (because it implies zero equal to zero). Thus equipartition of energy between gravitational and non-gravitational energy characterizes horizon.

\subsection{Kerr-Newmann black hole}

Following on the Reissner-Nordström black hole, we will now compute the Brown-York energy for the Kerr-Newman black hole and verify the veracity of the equipartition conjecture for the location of its horizon. It is described by the metric

$d s^{2}=-\left(\frac{\Delta-a^{2} \sin ^{2} \theta}{\rho^{2}}\right) d t^{2}-\frac{2 a \sin ^{2} \theta\left(r^{2}+a^{2}-\Delta\right)}{\rho^{2}} d t d \phi+\frac{\rho^{2}}{\Delta} d r^{2}+\rho^{2} d \theta^{2}+\frac{\Sigma}{\rho^{2}} \sin ^{2} \theta d \phi^{2}$

where we have defined the following quantities,

$$
\Delta=r^{2}+a^{2}+Q^{2}-2 M r ; \quad \rho^{2}=r^{2}+a^{2} \cos ^{2} \theta ; \quad \Sigma=\left(r^{2}+a^{2}\right)^{2}-\Delta a^{2} \sin ^{2} \theta
$$

where $M, Q$ and $a$ have the usual meaning as mass, charge and specific angular momentum of the black hole. The normalized normal to $r=$ constant surface within the $t=$ constant surface is spacelike, i.e., $n_{a} n^{a}=1$, which for the spacetime described by the above metric ansatz turns out to be,

$$
n_{\mu}=\left(\sqrt{\frac{\rho^{2}}{\Delta}}, 0,0\right) ; \quad n^{\mu}=\left(\sqrt{\frac{\Delta}{\rho^{2}}}, 0,0\right)
$$

Thus the extrinsic curvature of any $r=$ constant surface within the $t=$ constant hypersurface can be obtained as

$$
k=-\frac{1}{\sqrt{h}} \partial_{\mu}\left(\sqrt{h} n^{\mu}\right)=-\partial_{r} n^{r}-n^{r} \partial_{r} \ln \sqrt{h}
$$

The above expression nicely breaks into two parts, $\partial_{r} n^{r}$ and $\partial_{r} \ln \sqrt{h}$. Each of them can be evaluated individually leading to

$$
\partial_{r} n^{r}=\frac{1}{2} \sqrt{\frac{\Delta}{\rho^{2}}}\left(\frac{\partial_{r} \Delta}{\Delta}-\frac{\partial_{r} \rho^{2}}{\rho^{2}}\right) ; \quad \partial_{r} \ln \sqrt{h}=\frac{\partial_{r} \rho^{2}}{2 \rho^{2}}+\frac{\partial_{r} \Sigma}{2 \Sigma}-\frac{\partial_{r} \Delta}{2 \Delta}
$$

This immediately leads to the following expression for extrinsic curvature

$$
\begin{aligned}
k & =-\sqrt{\frac{\Delta}{\rho^{2}}} \frac{\partial_{r} \Sigma}{2 \Sigma} \\
& =-r \sqrt{1-\frac{2 M}{r}+\frac{a^{2}+Q^{2}}{r^{2}}} \frac{2 r\left(r^{2}+a^{2}\right)-(r-M) a^{2} \sin ^{2} \theta}{\sqrt{r^{2}+a^{2} \cos ^{2} \theta}\left[\left(r^{2}+a^{2}\right)^{2}-a^{2} \Delta \sin ^{2} \theta\right]}
\end{aligned}
$$


Now the metric on the two-surface is $\sqrt{q}=\sqrt{\Sigma} \sin \theta$. Hence the unreferenced Brown-York energy for the Kerr-Newmann black hole within a sphere of radius $r$ turns out to be

$$
\begin{aligned}
\mathcal{E} & =\frac{1}{8 \pi} \int d^{2} x \sqrt{q} k=\frac{1}{8 \pi} \int d \phi d \theta \sin \theta \sqrt{\Sigma} k \\
& =-\frac{1}{4} r \sqrt{1-\frac{2 M}{r}+\frac{a^{2}+Q^{2}}{r^{2}}} \int_{0}^{\pi} d \theta \sin \theta \frac{2 r\left(r^{2}+a^{2}\right)-(r-M) a^{2} \sin ^{2} \theta}{\sqrt{\left(r^{2}+a^{2} \cos ^{2} \theta\right)\left[\left(r^{2}+a^{2}\right)^{2}-a^{2} \Delta \sin ^{2} \theta\right]}}
\end{aligned}
$$

which in general cannot be integrated to obtain a closed form expression. However a closed form expression is indeed possible to obtain in the slow rotation limit i.e., $a / r \ll 1$. Then the above expression for unreferenced Brown-York energy reduces to [12]

$$
\begin{aligned}
\mathcal{E} & =-\frac{1}{2} r \sqrt{1-\frac{2 M}{r}+\frac{a^{2}+Q^{2}}{r^{2}}} \int_{0}^{\pi} d \theta \sin \theta\left[1-\frac{a^{2}}{2 r^{2}}\left\{\cos ^{2} \theta+\left(\frac{M}{r}-\frac{Q^{2}}{r^{2}}\right) \sin ^{2} \theta\right\}\right] \\
& =-r \sqrt{1-\frac{2 M}{r}+\frac{a^{2}+Q^{2}}{r^{2}}}\left[1-\frac{a^{2}}{6 r^{2}}\left(1+\frac{2 M}{r}-\frac{Q^{2}}{r^{2}}\right)\right]
\end{aligned}
$$

Getting the reference term is more difficult, for that we have to map the two dimensional surface to a flat three dimensional spacetime. If the flat three dimensional surface is described by the three coordinates $R, \vartheta, \Phi$, then the matching with two-surface would provide the following relations, $R=R(\theta), \vartheta=\vartheta(\theta)$ and $\Phi=\phi$. These relations have to be obtained through their substitution in the flat space line element and comparing with two-surface and Kerr-Newmann metric. This leads to the following differential equations for $R$ and $\vartheta$

$$
\begin{aligned}
& \left(\frac{d R}{d \theta}\right)^{2}+R^{2}\left(\frac{d \vartheta}{d \theta}\right)^{2}=\rho^{2} \\
& R^{2} \sin ^{2} \vartheta=\frac{\Sigma}{\rho^{2}} \sin ^{2} \theta
\end{aligned}
$$

We need to solve these two coupled differential equations to get both $R$ and $\vartheta$ in terms of $\theta$. Eq. (2.22) can be solved to get $R$ in terms of $\vartheta$ and $\theta$. This when substituted in eq. (2.21) would yield a differential equation of $\vartheta$. However the differential equation being complicated, in general (i.e., for arbitrary choices of the rotation parameter $a$ ) would not posses any analytic closed form solution. Thus to get analytic expression we need to use slow rotation limit, in which the solutions for $R(\theta)$ and $\vartheta(\theta)$ can be obtained as

$$
\begin{aligned}
& \sin \vartheta=\sin \theta\left[1+\frac{a^{2}}{2 r^{2}}\left(1+\frac{2 M}{r}-\frac{Q^{2}}{r^{2}}\right) \cos ^{2} \theta\right] \\
& R(\theta)=r\left[1+\frac{a^{2}}{2 r^{2}} \sin ^{2} \theta-\frac{a^{2}}{2 r^{2}}\left(\frac{2 M}{r}-\frac{Q^{2}}{r^{2}}\right) \cos ^{2} \theta\right]
\end{aligned}
$$

From these two relations we could obtain an expression for the extrinsic curvature $k_{0}$ of a two surface as embedded in flat space. Then this extrinsic curvature can be used to compute the reference term, which yields [12]

$$
\mathcal{E}_{0}=-r_{0}\left[1+\frac{a^{2}}{3 r_{0}^{2}}\left(1+\frac{M}{r_{0}}-\frac{Q^{2}}{2 r_{0}^{2}}\right)\right]
$$


It is interesting to note that there also appears the contribution of rotational energy in the flat space referenced energy. On expansion of $\mathcal{E}_{0}$ in powers of $1 / r$, the first order correction due to rotational energy is $-a^{2} / 3 r_{0}$, we will comment on it later on.

Using the expression for $\mathcal{E}$ from eq. (2.20) and the reference term as in eq. (2.25), the referenced Brown-York energy turns out to be [12]

$$
\begin{aligned}
& E\left(r \leq r_{0}\right) \equiv \mathcal{E}-\mathcal{E}_{0}=r_{0}\left[1-\sqrt{1-\frac{2 M}{r_{0}}+\frac{a^{2}+Q^{2}}{r_{0}^{2}}}\right]+\frac{a^{2}}{6 r_{0}}\left[2\left(1+\frac{M}{r_{0}}-\frac{Q^{2}}{2 r_{0}^{2}}\right)\right. \\
& \left.+\left(1+\frac{2 M}{r_{0}}-\frac{Q^{2}}{r_{0}^{2}}\right) \sqrt{1-\frac{2 M}{r_{0}}+\frac{Q^{2}}{r_{0}^{2}}}\right]
\end{aligned}
$$

which gives the quasilocal energy within a sphere of radius $r_{0}$. If we had taken the sphere of radius $r_{0}$ to infinity, it would as expected go to the ADM mass $M$. Expanding terms within square root in powers of $1 / r$ we arrive at

$$
E\left(r \leq r_{0}\right) \approx M-\frac{a^{2}+Q^{2}-M^{2}}{2 r_{0}}-\frac{M\left(a^{2}+Q^{2}\right)}{2 r_{0}^{2}}+\frac{a^{2}}{6 r_{0}}\left[3+\frac{3 M}{r_{0}}-\frac{2 M^{2}}{r_{0}^{2}}-2 \frac{Q^{2}}{r_{0}^{2}}+\frac{Q^{2}-M^{2}}{2 r_{0}^{2}}\right]
$$

Note that there is no contribution of rotational energy at order $1 / r_{0}$. This is precisely because rotational energy along with Kerr-Newmann spacetime, is also shared by the referenced spacetime. Thus as the reference term $\mathcal{E}_{0}$ is subtracted in order to get the Brown-York energy, the contribution from rotational part is exactly canceled. That is why the BrownYork energy for Kerr-Newmann spacetime is free of pure rotational terms and rotation only contributes through coupling with mass and charge. This is also the reason behind the fact that for the case of extremal black hole (i.e., $M^{2}=a^{2}+Q^{2}$ ) the Brown-York energy reduces to $M+\left(a^{2} / 2 r_{0}\right)$. Thus in addition to the ADM mass the rotational energy $a^{2} / 2 r_{0}$ also contributes to the Brown-York energy at large distance for extremal Kerr-Newmann black hole. Note that for $a=0$, we get back the result for extremal Reissner-Nordström black hole.

Now let's turn to our main aim of obtaining the location of horizon at the equipartition of gravitational and non-gravitational energy. The energy outside $r_{0}$ has the expression, $E\left(r \geq r_{0}\right)=E_{\text {grav }}+\left(Q^{2} / 2 r_{0}\right)$. Since total energy in the spacetime is the ADM mass $M$, we obtain the gravitational energy to be

$$
\begin{aligned}
E_{\text {grav }}=M & -\frac{Q^{2}}{2 r_{0}}-r_{0}\left[1-\sqrt{1-\frac{2 M}{r_{0}}+\frac{a^{2}+Q^{2}}{r_{0}^{2}}}\right]-\frac{a^{2}}{6 r_{0}}\left[2\left(1+\frac{M}{r_{0}}-\frac{Q^{2}}{2 r_{0}^{2}}\right)\right. \\
& \left.+\left(1+\frac{2 M}{r_{0}}-\frac{Q^{2}}{r_{0}^{2}}\right) \sqrt{1-\frac{2 M}{r_{0}}+\frac{Q^{2}}{r_{0}^{2}}}\right]
\end{aligned}
$$

On the other hand the non-gravitational energy is $E_{\text {non-grav }}=M-\left(a^{2}+Q^{2} / 2 r_{0}\right)$. Requiring equipartition of the two, i.e., gravitational and non-gravitational to be equal (with proper 
sign to ensure attractive nature of gravity), we finally obtain

$$
\begin{gathered}
\sqrt{1-\frac{2 M}{r_{0}}+\frac{a^{2}+Q^{2}}{r_{0}^{2}}}\left[\sqrt{1-\frac{2 M}{r_{0}}+\frac{a^{2}+Q^{2}}{r_{0}^{2}}}-1-\frac{a^{2}}{6 r_{0}^{2}} \sqrt{1-\frac{2 M}{r_{0}}+\frac{a^{2}+Q^{2}}{r_{0}^{2}}}\right. \\
\left.+\frac{a^{2}}{6 r_{0}^{2}}\left(1+\frac{2 M}{r_{0}}-\frac{Q^{2}}{r_{0}^{2}}\right)\right]=0
\end{gathered}
$$

This algebraic equation has two solutions: (a) $r=r_{+}=M+\sqrt{M^{2}-a^{2}-Q^{2}}$, the larger root of $r^{2}-2 M r+\left(Q^{2}+a^{2}\right)=0$ defining the horizon, and (b) $r=\left(a^{2}+Q^{2}\right) / 2 M$, the hard core radius for naked singularity, $a^{2}+Q^{2}>M^{2}$. Thus the equipartition conjecture is also verified for the Kerr-Newman black hole albeit in the slow rotation limit.

Having succeeded in deriving the horizon (or hard radius) location, starting from equipartition in the case of both charged and charged axi-symmetric configurations in general relativity it would be interesting to ask, what happens in higher dimensions, still in the premise of general relativity or more importantly, what happens to this equipartition conjecture when higher order curvature invariants are present in the action. That is what we take up in the next section.

\section{Brown-York quasilocal energy and black hole horizons in Lanczos- Lovelock gravity}

Before addressing the Brown-York energy for Lanczos-Lovelock gravity, as a warm up as well as to show the complexities involved, we will first discuss the case of Brown-York energy in higher dimension but within the context of general relativity. The Brown-York energy for a D-dimensional spacetime in general relativity is still given by the difference $k-k_{0}$ but this time evaluated for a $(D-2)$-dimensional surface. The geometry remains similar, the full manifold $\mathcal{M}$ is $D$-dimensional, in which we have constant time hypersurfaces, namely $\Sigma$, which are now $(D-1)$-dimensional and so is the surface $B$. Hence the boundary of $\Sigma$ as intersected by $B$ forms the desired co-dimension two-surface $\mathcal{B}$, which is now $(D-2)$ dimensional. However we need to account for proper volume factors. For this reason the Brown-York energy is written as

$$
E_{B Y}=\frac{1}{8 \pi \gamma} \int d A_{D-2}\left(k-k_{0}\right)
$$

where $\gamma$ is a numerical factor to be fixed later. Any static spherically symmetric spacetime can be presented by the metric eq. (2.2). The extrinsic curvature of the $(D-2)$-dimensional surface $\mathcal{B}$ is $k=-[(D-2) / r] \sqrt{g(r)}$ and $k_{0}=-(D-2) / r$. On substitution in eq. (3.1) and integration over the $(D-2)$-dimensional area immediately yields

$$
E_{B Y}=\frac{D-2}{8 \pi \gamma} \frac{2 \pi^{(D-1) / 2}}{\Gamma\left(\frac{D-1}{2}\right)} r^{D-3}(1-\sqrt{g(r)})
$$

By setting the constant $\gamma$ to the value

$$
\gamma=\frac{D-2}{\Gamma\left(\frac{D-1}{2}\right)} \frac{\pi^{(D-3) / 2}}{4}
$$


we obtain the following expression for Brown-York energy in a static spherically symmetric spacetime in general relativity in higher dimension

$$
E_{B Y}=r^{D-3}(1-\sqrt{g(r)})
$$

In $D$-dimensions static spherically symmetric solution of general relativity corresponds to, $f=g^{-1}=1-\left(M / r^{D-3}\right)$, which when substituted in the above expression, leads to $\lim _{r \rightarrow \infty} E_{B Y}=M$, i.e., the above expression agrees as required with ADM mass in the asymptotic limit.

However we cannot use this for Lanczos-Lovelock gravity. This can be seen directly as in $m$ th order Lanczos-Lovelock gravity the static spherically symmetric black hole solution is given by $f(r)=g(r)=1-\left(\gamma / r^{(D-(2 m+1)) / m}\right)$. In analogy with the Einstein case we write from eq. (3.4) the Brown-York energy as

$$
E_{B Y}=r^{\frac{D-2 m-1}{m}}\left[1-\left(1-\frac{\gamma}{r^{\frac{D-2 m-1}{m}}}\right)^{1 / 2}\right] \stackrel{r \rightarrow \infty}{=} \frac{\gamma}{2}
$$

which for large $r$ goes as $\gamma / 2$. It does not agree with the ADM mass which should really be $\propto \gamma^{m}$ [38]. However it is interesting that we get almost the right result but for mismatch in dimension of mass. This is of course not the right expression for quasi-local BrownYork energy, and we need to re-derive the Brown-York energy expression which would be appropriate for the Lanczos-Lovelock theory. That is what we will do next.

\subsection{Brown-York quasilocal energy in Lanczos-Lovelock gravity: formalism}

The Brown-York energy for Lanczos-Lovelock gravity can be obtained in a straightforward manner following the general relativity prescription. The derivation essentially amounts to calculate the counter term for gravitational action on a $t=$ constant surface (e.g., $\Sigma$ ) and the corresponding term for the $(D-2)$-boundary $\mathcal{B}$. Thus we can use the counter term for Lanczos-Lovelock gravity $[14,39-44]$ on the $t=$ constant hypersurface $\Sigma$ and then take it to the $(D-2)$-boundary $\mathcal{B}$. This leads to the following generalization of Brown-York energy to $m$ th order pure Lanczos-Lovelock gravity as,

$$
E_{B Y}^{(m)}=c^{(m)} \int d A_{D-2} \mathcal{E}
$$

where $c^{(m)}$ is a numerical factor which can be adjusted to get $E_{B Y}=M$ at infinity. In general relativity the quantity $\mathcal{E}$ was just $\left(k-k_{0}\right)$ and $c^{(1)}=\left(\Gamma\left(\frac{D-1}{2}\right)\right) /\left(2(D-2) \pi^{(D-1) / 2}\right)$, however for $m$ th order Lanczos-Lovelock gravity $\mathcal{E}$ turns out to be

$$
\mathcal{E}=\frac{m !}{2^{m+1}} \sum_{s=0}^{m-1} c_{s} \Pi^{(s)}
$$

where,

$$
\Pi^{(s)}=\delta_{B_{1} B_{2} \ldots B_{2 m-1}}^{A_{1} A_{2} \ldots A_{2 m-1}} R_{A_{1} A_{2}}^{B_{1} B_{2}} \cdots R_{A_{2 s-1} A_{2 s}}^{B_{2 s-1} B_{2 s}}\left(k_{A_{2 s+1}}^{B_{2 s+1}}-k_{(0) A_{2 s+1}}^{B_{2 s+1}}\right) \cdots\left(k_{A_{2 m-1}}^{B_{2 m-1}}-k_{(0) A_{2 m-1}}^{B_{2 m-1}}\right)
$$


Here $k_{(0) B}^{A}$ is the extrinsic curvature of the two surface as embedded in flat spacetime. Also the arbitrary constants $c_{s}$ appearing in eq. (3.7) has the following expression:

$$
c_{s}=\sum_{q=s}^{m-1} \frac{(-2)^{q-s} 4^{m-q}\left({ }^{q} C_{s}\right)}{q !(2 m-2 q-1) ! !}
$$

Here ${ }^{q} C_{s}$ is the combination symbol and stands for $q ! /\{(q-s) ! s !\}$. Before proceeding further let us pause for a while, and consider the case of static vacuum solution for $m$ th order pure Lanczos-Lovelock gravity which is given by [38, 45-47]

$$
d s^{2}=-f(r) d t^{2}+\frac{d r^{2}}{f(r)}+r^{2} d \Omega_{D-2}^{2} ; \quad f(r)=1-\left(\beta / r^{\frac{D-(2 m+1)}{m}}\right)
$$

It is clear that $\beta$ is a dimension full constant related to the ADM mass and $D$ is the dimension of the spacetime such that $D \geq 2 m+2$. Note that $D \geq 2 m+1$ ensures that the Lagrangian is not a topological term and $D=2 m+1$ is excluded because it represents a solid angle deficit that can only describe a global monopole [48] without black hole. The later computations would involve the curvature tensor components which are

$$
R_{i j}^{k l}=\frac{1}{r^{2}}(1-f) \delta_{i j}^{k l}
$$

However the ADM mass defined in the context of $m$ th order Lanczos-Lovelock gravity turns out to be $M=\hat{c} \beta^{m}$, where $\hat{c}$ is just a numerical constant. This numerical constant depends explicitly on the order of Lanczos-Lovelock Lagrangian and also on the coefficient of $\mathrm{m}$-th Lanczos-Lovelock Lagrangian, $c^{(m)}$. The exact value of $\hat{c}$ in this and subsequent examples would not hit us, since we will be concerned primarily with the structure of the results. Thus the acid test for our prescription of Brown-York energy as presented in eq. (3.6) would be to see whether it matches with the ADM mass $M$ as defined above.

In order to show the validity of our result, let us first see that it includes the general relativity case for $m=1$. We evaluate eq. (3.6) for $m=1$, which immediately leads to $s=0$. Using this in eq. (3.7) and eq. (3.8) we readily obtain, eq. (3.1), the correct quasilocal energy for general relativity.

Next we take up the Gauss-Bonnet action, which corresponds to $m=2$. Thus we have two possibilities $s=1$ and $s=0$ respectively. For $s=0$, we obtain

$$
\Pi^{(0)}=\delta_{B_{1} B_{2} B_{3}}^{A_{1} A_{2} A_{3}}\left(k_{A_{1}}^{B_{1}}-k_{(0) A_{2}}^{B_{2}}\right)\left(k_{A_{2}}^{B_{2}}-k_{(0) A_{2}}^{B_{2}}\right)\left(k_{A_{3}}^{B_{3}}-k_{(0) A_{3}}^{B_{3}}\right) \sim \frac{\beta^{3}}{r^{3} r^{3(D-5) / 2}}
$$

while for $s=1$, we get

$$
\Pi^{(1)}=\delta_{B_{1} B_{2} B_{3}}^{A_{1} A_{2} A_{3}} R_{A_{1} A_{2}}^{B_{1} B_{2}}\left(k_{A_{3}}^{B_{3}}-k_{(0) A_{3}}^{B_{3}}\right) \sim \frac{1}{r^{3}} \frac{\beta^{2}}{r^{D-5}}
$$

Then the Brown-York energy at large $r$ takes the form

$$
\begin{aligned}
E_{B Y} & =c^{(2)} r^{D-2}\left[c_{0} \frac{\beta^{3}}{r^{3} r^{3(D-5) / 2}}+c_{1} \frac{1}{r^{3}} \frac{\beta^{2}}{r^{D-5}}\right] \\
& =c^{(2)} c_{1} \beta^{2}+c^{(2)} c_{0} \beta^{3} r^{-\frac{D-5}{2}} \stackrel{r \rightarrow \infty}{=} \hat{c} \beta^{2}=M
\end{aligned}
$$


which exactly agrees with the ADM mass. Hence for pure Gauss-Bonnet gravity, the Brown-York energy at infinity exactly matches with the ADM mass.

It is now time to consider $m$ th order pure Lanczos-Lovelock gravity in which $s$ can take values $0,1, \ldots,(m-1)$ respectively. This immediately leads to the following expression

$$
\begin{aligned}
\Pi^{(0)} & =\delta_{B_{1} B_{2} \ldots B_{2 m-1}}^{A_{1} A_{2} \ldots A_{2 m-1}}\left(k_{A_{1}}^{B_{1}}-k_{(0) A_{2}}^{B_{2}}\right)\left(k_{A_{2}}^{B_{2}}-k_{(0) A_{2}}^{B_{2}}\right) \cdots\left(k_{A_{2 m-1}}^{B_{2 m-1}}-k_{(0) A_{2 m-1}}^{B_{2 m-1}}\right) \\
& \sim \frac{1}{r^{2 m-1}}(1-\sqrt{f})^{2 m-1} \stackrel{r \rightarrow \infty}{\longrightarrow} \frac{1}{r^{2 m-1}} \frac{\beta^{2 m-1}}{r^{(2 m-1)(D-2 m-1) / m}}
\end{aligned}
$$

Then,

$$
\begin{aligned}
\Pi^{(s)} & =\delta_{B_{1} B_{2} \ldots B_{2 m-1}}^{A_{1} A_{2} \ldots A_{2 m-1}} R_{A_{1} A_{2}}^{B_{1} B_{2}} \cdots R_{A_{2 s-1} A_{2 s}}^{B_{2 s-1} B_{2 s}}\left(k_{A_{2 s+1}}^{B_{2 s+1}}-k_{(0) A_{2 s+1}}^{B_{2 s+1}}\right) \cdots\left(k_{A_{2 m-1}}^{B_{2 m-1}}-k_{(0) A_{2 m-1}}^{B_{2 m-1}}\right) \\
& \sim \frac{1}{r^{2 m-1}}(1-f)^{s}(1-\sqrt{f})^{2 m-2 s-1} \stackrel{r \rightarrow \infty}{\longrightarrow} \frac{1}{r^{2 m-1}} \frac{\beta^{2 m-s-1}}{r^{(2 m-s-1)(D-2 m-1) / m}}
\end{aligned}
$$

and finally,

$$
\begin{aligned}
\Pi^{(m-1)} & =\delta_{B_{1} B_{2} \ldots B_{2 m-1}}^{A_{1} A_{2} \ldots A_{2 m-1}} R_{A_{1} A_{2}}^{B_{1} B_{2}} \cdots R_{A_{2 m-3} B_{2 m-3} B_{2 m-2}}^{B_{2 m-2}}\left(k_{A_{2 m-1}}^{B_{2 m-1}}-k_{(0) A_{2 m-1}}^{B_{2 m-1}}\right) \\
& \sim \frac{1}{r^{2 m-1}}(1-f)^{m-1}(1-\sqrt{f}) \stackrel{r \rightarrow \infty}{\longrightarrow} \frac{1}{r^{2 m-1}} \frac{\beta^{m}}{r^{D-2 m-1}}
\end{aligned}
$$

Hence the Brown-York energy at large $r$ turns out to have the following expression

$$
\begin{aligned}
\lim _{r \rightarrow \infty} E_{B Y}= & c^{(m)} r^{D-2}\left[c_{(0)} \frac{1}{r^{2 m-1}} \frac{\beta^{2 m-1}}{r^{(2 m-1)(D-2 m-1) / m}}+\cdots+c_{(s)} \frac{1}{r^{2 m-1}} \frac{\beta^{2 m-s-1}}{r^{(2 m-s-1)(D-2 m-1) / m}}\right. \\
& \left.+\cdots+c_{(m-1)} \frac{1}{r^{2 m-1}} \frac{\beta^{m}}{r^{D-2 m-1}}\right] \\
= & \hat{c} \beta^{m}+\cdots+\bar{c}_{(s)}\left(\beta^{2 m-s-1} / r^{(D-2 m-1)(m-s-1) / m}\right) \\
& +\cdots+\bar{c}_{(0)}\left(\beta^{2 m-1} / r^{(D-2 m-1)(m-1) / m}\right) \stackrel{r \rightarrow \infty}{=} M
\end{aligned}
$$

Thus we have proved that the Brown-York energy as defined by eq. (3.6) in the asymptotic limit leads to the ADM mass. Hence the definition of Brown-York energy works perfectly well and it passes the acid test of matching with ADM mass asymptotically for static black hole in Lanczos-Lovelock gravity. We have thus generalized the Brown-York energy for Lanczos-Lovelock gravity.

\subsection{Brown-York quasilocal energy in Lanczos-Lovelock gravity and equiparti- tion}

Having derived the quasilocal energy for Lanczos-Lovelock theories of gravity, we would now like to apply the equipartition conjecture to Lanczos-Lovelock gravity and verify its veracity. For any static spherically symmetric spacetime given by eq. (3.10) describing an $m$ th order Lovelock static black hole, the Brown-York energy reads as

$$
\begin{aligned}
E_{\mathrm{BY}}=r^{D-2 m-1}\left[\bar{c}_{(0)}(1-\sqrt{f})^{2 m-1}+\cdots+\bar{c}_{(s)}(1-\sqrt{f})^{2 m-2 s-1}(1-f)^{s}\right. \\
\left.+\cdots+\bar{c}_{m-1}(1-f)^{m-1}(1-\sqrt{f})\right]
\end{aligned}
$$


This is the energy, $E_{\mathrm{BY}}\left(r \leq r_{0}\right)$, lying inside radius $r=r_{0}$. Let us consider a charged black hole with

$$
f(r)=1-\left(\frac{\beta^{m}}{r^{D-2 m-1}}-\frac{\bar{Q}^{2}}{r^{2 D-2 m-4}}\right)^{1 / m}
$$

where $\beta^{m}=2 M / \bar{c}$ and $\bar{c}=\bar{c}_{(0)}+\cdots+\bar{c}_{(s)}+\cdots+\bar{c}_{(m)}$. For Maxwell field the energy outside $r=r_{0}$ is as before $E\left(r \geq r_{0}\right)=E_{\text {grav }}+\left(Q^{2} / 2 r_{0}^{D-3}\right)$ while non-gravitational component is $E_{\text {non-grav }}=M-\left(Q^{2} / 2 r_{0}^{D-3}\right)$. Requiring the total energy in spacetime to be equal to the ADM mass, we readily obtain the gravitational contribution to be,

$$
\begin{aligned}
E_{\text {grav }}=M & -\frac{Q^{2}}{2 r_{0}^{D-3}}-r_{0}^{D-2 m-1}\left[\bar{c}_{(0)}\left(1-\sqrt{f_{0}}\right)^{2 m-1}+\cdots+\bar{c}_{(s)}\left(1-\sqrt{f_{0}}\right)^{2 m-2 s-1}\left(1-f_{0}\right)^{s}\right. \\
& \left.+\cdots+\bar{c}_{m-1}\left(1-f_{0}\right)^{m-1}\left(1-\sqrt{f_{0}}\right)\right]
\end{aligned}
$$

where $f_{0}=f\left(r=r_{0}\right)$. Now the equipartition of gravitational and non-gravitational energy leads to the following algebraic equation

$$
\begin{aligned}
1 & -f_{0} \\
& =\left[\frac{\bar{c}_{(0)}\left(1-\sqrt{f_{0}}\right)^{2 m-1}+\cdots+\bar{c}_{(s)}\left(1-\sqrt{f_{0}}\right)^{2 m-2 s-1}\left(1-f_{0}\right)^{s}+\cdots+\bar{c}_{m-1}\left(1-f_{0}\right)^{m-1}\left(1-\sqrt{f_{0}}\right)}{\bar{c}_{(0)}+\cdots+\bar{c}_{(s)}+\cdots+\bar{c}_{(m)}}\right]^{1 / m}
\end{aligned}
$$

Note that the above equation has various powers of $f_{0}$ upto order $\mathrm{m}$, which is also the order of pure Lanczos-Lovelock Lagrangian. In general this is a complicated algebraic equation to solve for $f_{0}$. However we will avoid this difficulty by performing the following trick: we will substitute $f_{0}=0$ and $f_{0}=1$ in the above equation and see whether it is satisfied. As before it turns out that the above two indeed satisfy eq. (3.22). These two conditions have the two familiar solutions, one defining the horizon $r_{+}$, the larger root of $r^{2 D-2 m-4}-\beta^{m} r^{D-3}+\bar{Q}^{2}=0$ and the other, the hard core radius $r=\left(\bar{Q}^{2} / \beta^{m}\right)^{1 / D-3}$ for naked singularity, with $M^{2}<a^{2}+Q^{2}$. This is for the pure Lovelock analogue of Reissner-Nordström black hole which includes for $Q=0$ the pure Lovelock analogue of the Schwarzschild black hole. Thus we verify that the equipartition conjecture continues to hold good for pure Lanczos-Lovelock static black holes.

The important point to note is that the conjecture turns out to hold good only for pure Lovelock (for a fixed $m$ ) black holes but not for Einstein-Lovelock (with sum over $m$ ) black holes. This is what we show next for the case of Einstein-Gauss-Bonnet black hole.

\subsection{Equipartition conjecture in Einstein-Gauss-Bonnet gravity}

In the previous section we have concentrated on the pure Lovelock theories and the validity of equipartition conjecture. In this section we will illustrate that Equipartition conjecture does not hold for the general Lovelock theories. For that we will use an action, which is sum of the Einstein-Hilbert and Gauss-Bonnet terms. The static black hole solution corresponding to the Einstein-Gauss-Bonnet action, is known as the Boulware-Deser solution [49, 50], it reads as follows:

$$
d s^{2}=-f(r) d t^{2}+\frac{d r^{2}}{f(r)}+r^{2} d \Omega_{3}^{2} ; \quad f(r)=1-\frac{r^{2}}{2 \alpha}\left[-1 \pm \sqrt{1+\frac{4 \alpha M}{r^{4}}}\right]
$$


Here $\alpha$ is the Gauss-Bonnet coupling constant and $M$ is the mass term, and both of them are of dimension $L^{2}$. There are two branches of the solution, the one with +ve sign has the right Einstein limit with attractive gravity while the other (i.e., the one with - ve sign) is repulsive. We would therefore choose the former. At $r=0$ we have a curvature singularity, which is cloaked by an event horizon for the +ve branch for $M \geq \alpha$ and is located at, $r^{2}=r_{h}^{2}=M-\alpha$. Otherwise the above solution would represent a naked singularity. Let us now compute the Brown-York energy for the Einstein-Gauss-Bonnet solution. For which the quasi-local energy for $D=5$ involves both $m=1$, the Einstein-Hilbert term and the $m=2$, the Gauss-Bonnet term. For this, from eq. (3.4) and eq. (3.19) we write

$$
E\left(r \leq r_{0}\right)=r_{0}^{2}\left(1-\sqrt{f_{0}}\right)+\alpha\left[c_{1}\left(1-\sqrt{f_{0}}\right)^{3}+c_{2}\left(1-f_{0}\right)\left(1-\sqrt{f_{0}}\right)\right]
$$

where $c_{1}$ and $c_{2}$ are two constants whose values can be obtained from eq. (3.9) and $f_{0}=$ $f\left(r=r_{0}\right)$. As an illustration we can consider the asymptotic limit of the Brown-York energy defined in eq. (3.24). For which the first term yields $r_{0}^{2} \times\left(M / r_{0}^{2}\right)$, while the second term leads to, $M^{3} / r_{0}^{6}+M^{2} / r_{0}^{4}$. Thus in the asymptotic limit, i.e., $r_{0} \rightarrow \infty$ Brown-York energy exactly equals the ADM mass $M$.

Given the Brown-York energy, the gravitational energy is just the difference, $M-E(r \leq$ $\left.r_{0}\right)$, and non-gravitational energy is anyway the ADM mass $M$. Then the equipartition demands

$$
2 M=r_{0}^{2}\left(1-\sqrt{f_{0}}\right)+\alpha\left[c_{1}\left(1-\sqrt{f_{0}}\right)^{3}+c_{2}\left(1-f_{0}\right)\left(1-\sqrt{f_{0}}\right)\right]
$$

Clearly this does not define horizon for the Einstein-Gauss-Bonnet black hole. For horizon $f\left(r_{0}\right)=0$ which gives $2 M=r_{h}^{2}+\alpha\left(c_{1}+c_{2}\right)$. This cannot be satisfied because $c_{1}, c_{2}$ are numerical factors. The equipartition conjecture therefore does not work for Einstein-GaussBonnet and in general for Einstein-Lovelock black holes. It works only for pure Lovelock black holes.

This explicitly shows the special status of pure Lanczos-Lovelock gravity as also exposed in [19-21]. In this case as well only for pure Lovelock black holes, the equipartition conjecture holds good and defines the horizon. Thus equipartition conjecture discerns the pure Lanczos-Lovelock gravity from the general Einstein-Lanczos-Lovelock gravity.

\section{Discussion}

One of the promising candidates for obtaining quasilocal energy in general relativity is the Brown-York energy. This definition is based on the Hamilton-Jacobi treatment of the gravitational action written in terms of $\mathrm{ADM}$ variables and then identifying the correct expression for energy. The salient feature of this prescription is that, at asymptotic infinity it correctly reproduces the ADM mass, which acts as an acid test for any definition of energy in general relativity. In this work we have generalized the Brown-York energy for Lanczos-Lovelock gravity and have employed it to verify the veracity of the equipartition conjecture for defining black hole horizon. That is horizon marks equality of gravitational and non-gravitational energy. 
It is envisioned that when a configuration is infinitely dispersed, it has energy equal to ADM mass $M$ as it begins collapsing under its own gravity, it picks up gravitational energy which is negative, and electric field and rotational energy for a charged and rotating black hole. Thus at any finite $r$, there are gravitational and non-gravitational components of energy. The Brown-York energy gives energy contained inside a given radius, from which if we subtract non-gravitational part, we can compute gravitational energy. This could be done for static black holes and for axially symmetric Kerr-Newmann black hole it can be evaluated in the slow rotation limit. By means of the Brown-York energy expression, we can tame the notorious gravitational field energy to obtain a quantitative expression. The interesting application of which was made by one of us in proposing the equipartition conjecture [10] for characterization of black hole horizon by equality of gravitational and non-gravitational energy. It is motivated by the fact that as horizon is approached timelike particles tend to null particles. Motion of the former is governed by $\nabla \Phi$ produced by nongravitational energy while that of the latter by spatial curvature caused by gravitational energy [10]. Thus as the former approaches the latter at the horizon so should be their sources. Thus gravitational and non-gravitational energy must be equal at the horizon.

We have verified the equipartition conjecture for static and axially symmetric (in the slow rotation limit) black holes, in particular Kerr-Newmann black hole that includes static black holes. It is remarkable that for extremal Reissner-Nordström black hole gravitational and electric field energy exactly cancel out each-other everywhere so that the Brown-York energy is conserved mass $M$.

It turns out that the definition of Brown-York energy cannot straightway be taken over to Lanczos-Lovelock gravity but it needs to be supplemented with the counter terms. With this modification, we generalize the Brown-York energy expression for Lanczos-Lovelock gravity and again separate out gravitational and non-gravitational parts. We use that to establish the equipartition conjecture for static pure Lovelock black holes. It is interesting that the conjecture holds good only for pure (for a fixed $m$ ) Lovelock but not for general (sum over $m$ ) Einstein-Lovelock black holes. Like some other features [19-21], the equipartition of gravitational and non-gravitational energy defining the black hole horizon is also yet another discriminator of pure Lovelock gravity.

\section{Acknowledgments}

Research of S.C. is funded by a SPM fellowship from CSIR, Government of India. A part of the work was done while ND was visiting Albert Einstein Institute, Golm and University of KwaZulu-Natal, Durban, and for that he thanks respectively Hermann Nicolai and Sunil Maharaj.

Open Access. This article is distributed under the terms of the Creative Commons Attribution License (CC-BY 4.0), which permits any use, distribution and reproduction in any medium, provided the original author(s) and source are credited. 


\section{References}

[1] A. Komar, Covariant conservation laws in general relativity, Phys. Rev. 113 (1959) 934 [INSPIRE].

[2] S. Hawking, Gravitational radiation in an expanding universe, J. Math. Phys. 9 (1968) 598 [INSPIRE].

[3] R. Penrose, Quasilocal mass and angular momentum in general relativity, Proc. Roy. Soc. Lond. A 381 (1982) 53 [InSPIRE].

[4] A.J. Dougan and L.J. Mason, Quasilocal mass constructions with positive energy, Phys. Rev. Lett. 67 (1991) 2119 [inSPIRE].

[5] M. Ludvigsen and J.A.G. Vickers, The positivity of the Bondi mass, J. Phys. A 14 (1981) L389 [INSPIRE].

[6] R. Kulkarni, V. Chellathurai and N. Dadhich, The effective mass of the Kerr spacetime, Class. Quant. Grav 5 (1988) 1443.

[7] G. Bergqvist, Quasilocal mass for event horizons, Class. Quant. Grav. 9 (1992) 1753.

[8] J.D. Brown and J.W. York, Jr., Quasilocal energy and conserved charges derived from the gravitational action, Phys. Rev. D 47 (1993) 1407 [gr-qc/9209012] [INSPIRE].

[9] N. Dadhich, On the Schwarzschild field, gr-qc/9704068 [INSPIRE].

[10] N. Dadhich, Black hole: equipartition of matter and potential energy, Curr. Sci. 76 (1999) 831 [gr-qc/9705037] [INSPIRE].

[11] S. Bose and N. Dadhich, On the Brown-York quasilocal energy, gravitational charge and black hole horizons, Phys. Rev. D 60 (1999) 064010 [gr-qc/9906063] [INSPIRE].

[12] S. Bose and T.Z. Naing, Quasilocal energy for rotating charged black hole solutions in general relativity and string theory, Phys. Rev. D 60 (1999) 104027 [hep-th/9911070] [INSPIRE].

[13] T. Padmanabhan, Gravitation: foundations and frontiers, Cambridge University Press, Cambridge U.K. (2010).

[14] T. Padmanabhan and D. Kothawala, Lanczos-Lovelock models of gravity, Phys. Rept. 531 (2013) 115 [arXiv:1302.2151] [INSPIRE].

[15] N. Dadhich, J.M. Pons and K. Prabhu, On the static Lovelock black holes, Gen. Rel. Grav. 45 (2013) 1131 [arXiv:1201.4994] [INSPIRE].

[16] J.M. Pons and N. Dadhich, On static black holes solutions in Einstein and Einstein-Gauss-Bonnet gravity with topology $S^{n} \times S^{n}$, Eur. Phys. J. C 75 (2015) 280 [arXiv: 1408.6754] [INSPIRE].

[17] N. Dadhich and J.M. Pons, Static pure Lovelock black hole solutions with horizon topology $S^{(n)} \times S^{(n)}$, JHEP 05 (2015) 067 [arXiv: 1503.00974] [INSPIRE].

[18] N. Dadhich, Characterization of the Lovelock gravity by Bianchi derivative, Pramana 74 (2010) 875 [arXiv:0802.3034] [INSPIRE].

[19] N. Dadhich, A discerning gravitational property for gravitational equation in higher dimensions, arXiv: 1506.08764 [INSPIRE].

[20] N. Dadhich, S.G. Ghosh and S. Jhingan, The Lovelock gravity in the critical spacetime dimension, Phys. Lett. B 711 (2012) 196 [arXiv:1202.4575] [INSPIRE]. 
[21] N. Dadhich, The gravitational equation in higher dimensions, Springer Proc. Phys. 157 (2014) 43 [arXiv: 1210.3022] [INSPIRE].

[22] A. Yale and T. Padmanabhan, Structure of Lanczos-Lovelock Lagrangians in critical dimensions, Gen. Rel. Grav. 43 (2011) 1549 [arXiv:1008.5154] [INSPIRE].

[23] T. Padmanabhan, Thermodynamical aspects of gravity: new insights, Rept. Prog. Phys. 73 (2010) 046901 [arXiv:0911.5004] [INSPIRE].

[24] T. Padmanabhan, General relativity from a thermodynamic perspective, Gen. Rel. Grav. 46 (2014) 1673 [arXiv: 1312.3253] [INSPIRE].

[25] S. Chakraborty and T. Padmanabhan, Evolution of spacetime arises due to the departure from holographic equipartition in all Lanczos-Lovelock theories of gravity, Phys. Rev. D 90 (2014) 124017 [arXiv: 1408.4679] [INSPIRE].

[26] S. Chakraborty and T. Padmanabhan, Geometrical variables with direct thermodynamic significance in Lanczos-Lovelock gravity, Phys. Rev. D 90 (2014) 084021 [arXiv:1408.4791] [INSPIRE].

[27] S. Chakraborty, Lanczos-Lovelock gravity from a thermodynamic perspective, JHEP 08 (2015) 029 [arXiv : 1505.07272] [INSPIRE].

[28] S. Chakraborty and T. Padmanabhan, Thermodynamical interpretation of the geometrical variables associated with null surfaces, Phys. Rev. D 92 (2015) 104011 [arXiv:1508.04060] [INSPIRE].

[29] R.L. Arnowitt, S. Deser and C.W. Misner, The dynamics of general relativity, Gen. Rel. Grav. 40 (2008) 1997 [gr-qc/0405109] [INSPIRE].

[30] E. Poisson, A relativist's toolkit: the mathematics of black-hole mechanics, $1^{\text {st }}$ ed., Cambridge University Press, Cambridge U.K. (2007).

[31] Y. Shi and L.-F. Tam, Positive mass theorem and the boundary behaviors of compact manifolds with nonnegative scalar curvature, J. Diff. Geom. 62 (2002) 79 [math/0301047].

[32] X.-Q. Fan, Y. Shi and L.-F. Tam, Large-sphere and small-sphere limits of the Brown-York mass, Commun. Anal. Geom. 17 (2009) 37 [arXiv:0711.2552].

[33] N.O. Murchadha, R.-S. Tung, N. Xie and E. Malec, The Brown-York mass and the Thorne hoop conjecture, Phys. Rev. Lett. 104 (2010) 041101 [arXiv:0912.4001] [INSPIRE].

[34] E. Malec and N. Xie, Brown-York mass and the hoop conjecture in nonspherical massive systems, Phys. Rev. D 91 (2015) 081501 [arXiv:1503.01354] [inSPIRE].

[35] S. Chakraborty and S. Chakraborty, Trajectory around a spherically symmetric non-rotating black hole, Can. J. Phys. 89 (2011) 689 [arXiv:1109.0676] [INSPIRE].

[36] S. Chakraborty, Aspects of neutrino oscillation in alternative gravity theories, JCAP 10 (2015) 019 [arXiv : 1506. 02647] [INSPIRE].

[37] S. Chakraborty and S. SenGupta, Solar system constraints on alternative gravity theories, Phys. Rev. D 89 (2014) 026003 [arXiv:1208.1433] [INSPIRE].

[38] D. Kastor, Komar integrals in higher (and lower) derivative gravity, Class. Quant. Grav. 25 (2008) 175007 [arXiv:0804.1832] [INSPIRE].

[39] G. Kofinas and R. Olea, Universal regularization prescription for Lovelock AdS gravity, JHEP 11 (2007) 069 [arXiv:0708.0782] [InSPIRE]. 
[40] O. Mišković and R. Olea, Counterterms in dimensionally continued AdS gravity, JHEP 10 (2007) 028 [arXiv:0706.4460] [INSPIRE].

[41] C. Barrabes and W. Israel, Lagrangian brane dynamics in general relativity and Einstein-Gauss-Bonnet gravity, Phys. Rev. D 71 (2005) 064008 [gr-qc/0502108] [INSPIRE].

[42] Y. Brihaye and E. Radu, Black objects in the Einstein-Gauss-Bonnet theory with negative cosmological constant and the boundary counterterm method, JHEP 09 (2008) 006 [arXiv:0806.1396] [INSPIRE].

[43] Y. Brihaye and E. Radu, Black hole solutions in D = 5 Chern-Simons gravity, JHEP 11 (2013) 049 [arXiv: 1305.3531] [INSPIRE].

[44] Y. Brihaye, B. Kleihaus, J. Kunz and E. Radu, Rotating black holes with equal-magnitude angular momenta in D = 5 Einstein-Gauss-Bonnet theory, JHEP 11 (2010) 098 [arXiv:1010.0860] [INSPIRE].

[45] J. Crisostomo, R. Troncoso and J. Zanelli, Black hole scan, Phys. Rev. D 62 (2000) 084013 [hep-th/0003271] [INSPIRE].

[46] S. Deser and B. Tekin, Gravitational energy in quadratic curvature gravities, Phys. Rev. Lett. 89 (2002) 101101 [hep-th/0205318] [INSPIRE].

[47] A. Baykal, Energy definition for quadratic curvature gravities, Phys. Rev. D 86 (2012) 127501 [arXiv: 1212.0432 ] [INSPIRE].

[48] M. Barriola and A. Vilenkin, Gravitational field of a global monopole, Phys. Rev. Lett. 63 (1989) 341 [INSPIRE].

[49] D.G. Boulware and S. Deser, String generated gravity models, Phys. Rev. Lett. 55 (1985) 2656 [INSPIRE].

[50] J.T. Wheeler, Symmetric solutions to the Gauss-Bonnet extended Einstein equations, Nucl. Phys. B 268 (1986) 737 [INSPIRE]. 